\title{
Máscara termoplástica recubierta de silicona para el manejo de la cicatriz hipertrófica facial
}

\author{
Jorge Andrés Rueda-Gutiérrez* \\ Karen Rodríguez- Franco** \\ Sandra Yohana Quintero - Moya*** \\ Mercedes Gutiérrez - Solano**** \\ Genny Liliana Meléndez - Flórez******
}

\begin{abstract}
* Médico y cirujano. Pontificia Universidad Javeriana. Estudiante de Cuarto año de Posgrado de Cirugía Plástica, Estética y Reconstructiva. Universidad Industrial de Santander. Bucaramanga. Colombia.

** Médica y Cirujana. Universidad Industrial de Santander. Universidad Industrial de Santander. Bucaramanga. Colombia.

***Fisioterapeuta Unidad de Quemados Hospital Universitario de Santander. Docente Universidad de Santander. Bucaramanga. Colombia.

****Fisioterapeuta Unidad de Quemados Hospital Universitario de Santander. Bucaramanga. Colombia.

*****Cirujana Plástica. Estética y Reconstructiva de la Universidad Nacional Autónoma de México. Máster en Microcirugía Universidad Autónoma de Barcelona. Especialista en Docencia y Profesora de Planta Universidad Industrial de Santander. Bucaramanga. Colombia.

Correspondencia:Dr. Jorge Rueda. Dirección: Carrera 26 No. 35-170. Conjunto Residencial Altos de Cañaveral V Etapa, Floridablanca. Colombia. Teléfono: (+057) 3204019674. Correo electrónico: Jorge.rueda51@hotmail.com.
\end{abstract}

Resumen

Las quemaduras secundarias a agresión física con intención de desfigurar, torturar o incluso asesinar, se han convertido en un motivo de consulta común en el siglo XXI, siendo Bangladesh el país con la más alta incidencia en el mundo. Colombia es uno de los países con mayor incidencia a nivel de Latinoamérica. La mayoría de las lesiones ocurre en áreas expuestas como el rostro y se acompaña de graves secuelas físicas, estéticas y funcionales. Se realiza la presentación de caso de una paciente de 35 años con quemaduras de tercer grado en región frontal, periocular, malar bilateral, nasal, labial superior y pabellón auricular derecho, manejada con injertos de piel, quien posteriormente desarrolla cicatriz hipertrófica. Se describe el tratamiento con máscara termoplástica elaborada por los profesionales tratantes, con cubierta interna de silicona, fabricada sobre molde a medida y ajustada con bandas elásticas; integrando en un único dispositivo removible, cómodo y de bajo costo, diferentes alternativas terapéuticas que logran modular efectivamente el proceso de cicatrización y por su simplicidad favorecen la adherencia al tratamiento, la cual es indispensable para obtener resultados satisfactorios. MED.UIS.2020;33(3): 49-58.

Palabras clave: Cicatriz Hipertrófica. Cicatrización de Heridas. Elastómeros de Silicona. Presión. Injerto de piel.

\section{Silicone coated thermoplastic mask for management of hypertrophic facial scars}

Abstract

Burns secondary to physical aggression with the intention of disfiguring, torturing or even murdering, have become a common reason for consultation in the 21st century, with Bangladesh being the country with the highest incidence in the world. Colombia is one of the countries with the highest incidence in Latin America. Most injuries occur in exposed areas such as the face and are accompanied by serious physical, aesthetic and functional sequelae. We present the case of a 35-year-old patient with third degree burns in the frontal, periocular, bilateral malar, nasal, upper labial and right ear region, managed with skin grafts, who later developed a hypertrophic scar. The treatment with a thermoplastic mask made by the treating professionals is described, with an internal silicone cover, made on a custom mold and adjusted with elastic bands; integrating in a single removable, comfortable and low-cost device, different therapeutic alternatives that manage to effectively modulate the healing process and, due to their simplicity, favor adherence to treatment, which is essential to obtain satisfactory results. MED.UIS.2020;33(3): 49-58.

Keywords: Hypertrophic Scar. Wound Healing. Silicone Elastomers. Pressure. Skin Graft 
¿Cómo citar este artículo?: Rueda - Gutiérrez JA, Rodríguez- Franco K, Quintero - Moya SL, Gutiérrez - Solano M, Meléndez - Flórez G. Máscara termoplástica recubierta de silicona para el manejo de la cicatriz hipertrófica facial. MED.UIS.2020;33(3):49-58. doi: 10.18273/revmed.v33n32020006

\section{Introducción}

Los ataques con ácido o líquido hirviente, con el fin de mutilar, desfigurar, torturar o incluso asesinar, se han convertido en una forma de agresión que ha aumentado de forma vertiginosa durante lo que va del siglo XXI, siendo Bangladesh el país con la más alta incidencia en el mundo y las mujeres el $80 \%$ de las víctimas ${ }^{1}$.Este lugar en la estadística condujo a la creación de la Fundación para los Sobrevivientes de Ácido en ese país (ASF, por sus siglas en inglés), con la cual se brinda apoyo a las víctimas, desde el tratamiento inicial de la quemadura hasta la rehabilitación médica y social. Otros países que reportan altas tasas de incidencia son Camboya, India, Pakistán y Uganda. En algunos países del continente africano se ha reportado este tipo de ataque violento como arma de guerra ${ }^{2}$. En países como Estados Unidos y Reino Unido, los ataques se relacionan con minorías étnicas, xenofobia, uso de drogas y en menor medida como violencia de género. Uno de los aspectos relevantes de este tipo de arma es que es usada con mayor frecuencia contra la mujer o como ataque homofóbico, es barata, de fácil acceso, se acompaña de alta tasa de impunidad y deja secuelas permanentes 3 . Colombia es uno de los países con mayor incidencia a nivel de Latinoamérica y se reporta una tasa hombre:mujer de 1:304. Por esta razón recientemente una víctima lideró un movimiento que condujo a la creación de una ley conocida como la "Ley Natalia Ponce" con la cual se incrementan las penas y se impone además castigo económico al perpetrador 5 .

Un estudio reciente reporta una incidencia en Colombia de $53,7 \%$ de quemaduras secundarias a agresión física a mujeres ${ }^{6}$. Las áreas afectadas con mayor frecuencia son las zonas anatómicas expuestas como el rostro, cuello, tórax y miembros superiores. En comparación con otras etiologías, hasta un $30 \%$ de los pacientes con quemaduras severas experimentan algún grado de hipertrofia de las cicatrices tanto de las quemaduras epitelizadas, como de las zonas tratadas con injertos y colgajos7; esto significa que las cicatrices después de la quemadura por ácido en cara, cuello y tórax, en donde el agresor busca deformar los senos y los complejos areola-pezón, son estigmatizantes y suficientemente deformantes como para lesionar permanentemente a la víctima tanto física como emocionalmente. Es por esta razón que hemos decidido presentar este caso y realizar una revisión bibliográfica del tema, en busca de difundir la patología y la situación médica y social, buscando concientizar a la comunidad médica y sobre todo sensibilizarla, a fin de que los pacientes reciban el tratamiento adecuado, en el lugar adecuado, teniendo en cuenta todas las consideraciones del caso.

Las cicatrices de la piel son el resultado de la respuesta inflamatoria después de una lesión dérmica. El proceso de cicatrización de la piel es dinámico y tiene como objetivo restablecer la función de barrera de la piel y las características físicas y mecánicas de protección evitando pérdida en la elasticidad de los tejidos circundantes, favoreciendo la recuperación de los arcos de movilidad en articulaciones, conservando las funciones específicas de estructuras especiales como los párpados o las áreas de mímica facial y generando un resultado estético favorable. El proceso de cicatrización tiene una duración de 1 año o más, y se divide en cuatro fases que se superponen entre sí:

Fase de Coagulación: Ocurre de manera inmediata después de la injuria y tiene el objetivo de detener la hemorragia. Se caracteriza por vasoconstricción refleja y activación de la cascada de la coagulación, generando un coagulo por la unión de fibras de fibrina y migración plaquetaria, con la mediación de interleucina (IL -6 e IL-8) y quimiocinas ${ }^{8}$.

Fase Inflamatoria: Inicia con el periodo inflamatorio temprano, se produce desde el primero hasta el tercer día. Durante este periodo las citoquinas proinflamatorias como el factor de crecimiento transformante $\mathrm{B}$, factor de crecimiento derivado de plaquetas y de crecimiento endotelial, estimulan la migración y activación de neutrófilos para eliminar el tejido desvitalizado y neutralizar patógenos. El periodo inflamatorio tardío se produce desde las primeras 48 horas hasta la primera semana de la injuria. Se caracteriza por la fagocitosis a cargo de los macrófagos, quienes se encargan de encapsular detritos creando un ambiente que previene la infección. Concomitantemente se activa 
Septiembre - diciembre

el complemento por acción de IL1 y el factor de necrosis tumoral ${ }^{8,9}$.

Fase Proliferativa: se produce entre el día 3 y el día 14, consta de 4 procesos, el proceso de fibroplastia, en el cual los fibroblastos se encargan de la producción de componentes de la matriz extracelular, inicialmente producen colágeno tipo III, y en menor medida tipo I y IV, así como glucosaminoglicanos, proteoglicanos y ácido hialurónico; el proceso de granulación caracterizado por la angiogénesis, el proceso de contracción en el cual los miofibroblastos disminuyen las dimensiones de la herida y finalmente el proceso de epitelización, dado por el crecimiento vertical de queratinocitos. La regulación de la producción y apoptosis de fibroblastos y miofibroblastos, así como la producción de colágeno y de los componentes de la matriz extracelular, está mediada por el factor de crecimiento transformante beta (TGF- $\beta$ ), factor de crecimiento derivado de plaquetas y factor de crecimiento endotelial, entre otros ${ }^{10,1,112}$.

Fase de Remodelación: Ocurre entre la segunda semana y hasta doce meses después de la lesión, durante esta fase se reemplazan las fibras de colágeno tipo III por colágeno tipo I aumentando progresivamente la fuerza tensil del tejido, produciendo una cicatriz definitiva, organizada y funcional, con un $80 \%$ de fuerza tensil respecto a la piel sana ${ }^{13}$.

Es necesario el equilibrio entre la replicación y la apoptosis de las células que participan en la producción de los nuevos elementos de la matriz extracelular durante el proceso de cicatrización. Este equilibrio puede ser afectado por múltiples factores dependientes del individuo, como la edad, predisposición genética, raza, niveles hormonales, respuesta inmunológica y estado nutricional; y por factores específicos de la herida como el mecanismo del trauma, dimensiones de la herida, profundidad, ubicación anatómica, tensión a la que están expuestas las lesiones, presencia de infección y tratamientos aplicados ${ }^{10,11,14}$.

Bajo circunstancias normales, la producción de TGF- $\beta$ disminuye al finalizar la fase proliferativa y durante el inicio de la fase de remodelación, sin embargo, se ha demostrado en modelos celulares, que en procesos de cicatrización hipertrófica y queloide persiste la expresión deTGF-ß̧yseperpetúan las fasesinflamatoria y proliferativa de la cicatrización ${ }^{15}$. Adicionalmente, la permanencia de células inflamatorias y factores
Máscara termoplástica recubierta de silicona para el manejo de la cicatriz hipertrófica facial

proinflamatorios, como la interleucina (IL) -1a, IL-1 $\beta$, IL-6 y el factor de necrosis tumoral $\alpha$ están regulados positivamente, causando crecimiento invasivo y desmedido de estas cicatrices ${ }^{13,15}$.

El colágeno que se produce inicialmente durante la fase proliferativa de la cicatrización es principalmente de tipo III, que por su estructura es menos funcional y elástico que el colágeno tipo $\mathrm{I}^{13}$. En una cicatriz hipertrófica, estos haces de colágeno tipo III se producen entre 3 y 20 veces más que en una cicatriz normal ${ }^{9}$, se disponen en paralelo a la superficie epidérmica con un patrón ondulado, generando inclusiones y nódulos que contienen miofibroblastos, disminuyendo así su predisposición a la muerte celular programada, provocando contracturas y liberando la producción exagerada de colágeno tipo I durante la fase de remodelación, cuya síntesis es siete veces más alta que en una cicatriz normal ${ }^{16,17}$.

Las cicatrices hipertróficas pueden aparecer desde las primeras 2 semanas del trauma y se manifiestan con mayor frecuencia entre la quinta y octava semana ${ }^{18,19}$. Se caracterizan por presentar un crecimiento exuberante de los tejidos, generando cambios estéticos que afectan la apariencia, la funcionalidad, la salud mental y por tanto la calidad de vida ${ }^{9,15,16}$.

Se ha descrito una mayor tasa de cicatrización hipertrófica en población joven, entre 12 a 30 años, debido probablemente al uso de dispositivos estéticos, perforaciones corporales, tatuajes y a que les dan mayor importancia a las cicatrices, consultando con mayor frecuencia. Adicionalmente, este grupo poblacional presenta una mayor tasa de síntesis de colágeno, incrementando la formación de tejido cicatrizal y una mayor cantidad de fibras elásticas en la piel que generan tensión sobre las cicatrices. Después de los 65 años es infrecuente encontrar cicatrices queloides o hipertróficas. En cuanto a la raza, se ha visto mayor predisposición en poblaciones asiáticas y en la raza negra, quienes tienen un riesgo 15 veces mayor de desarrollar queloides en comparación con la población de raza blanca ${ }^{17,20}$.

Anualmente 100 millones de personas en el continente americano desarrollan cicatrices secundarias a procedimientos quirúrgicos electivos o por trauma y se estima que hasta el $15 \%$ de estas personas puedan desarrollar procesos patológicos de cicatrización ${ }^{20}$.

La evaluación clínica de las cicatrices se realiza con la aplicación de escalas que buscan objetivizar las 
Rueda-Gutiérrez JA, Rodríguez-Franco K, Quintero-Moya SL, Gutiérrez-Solano M, Meléndez - Flórez G.

características de las cicatrices con el fin de realizar diagnóstico, hacer seguimiento clínico y evaluar respuesta a los tratamientos realizados, una de las escalas aplicadas con mayor frecuencia es la de Vancouver, que asigna diferentes puntajes según el grado de pigmentación, vascularidad, flexibilidad, volumen y presencia de contracturas. No existe consenso sobre a partir de que puntaje se considera una cicatriz como hipertrófica, sin embargo, esta escala es especialmente útil para evaluar la evolución de las cicatrices a través del tiempo ${ }^{21,22}$.

Una vez se identifica una alteración en el proceso de cicatrización, se debe establecer un tratamiento temprano y acorde a la lesión. Existen múltiples guías de tratamiento, que incluyen el masaje de la cicatriz, presión local, uso de bandas de silicona, infiltración intralesional de esteroides, crioterapia, resección quirúrgica, medicamentos biológicos tópicos, resección quirúrgica combinado con radioterapia postoperatoria, entre otros; cada uno con niveles de evidencia variables y con indicaciones específicas para su aplicación ${ }^{9,23}$. Las láminas de silicona y la presoterapia han sido ampliamente estudiadas en la última década. Bao Y. y cols., realizaron un metaanálisis en población asiática, donde evalúan el uso y efectividad de alternativas para el manejo de lesiones por quemaduras, dentro de las que se ha hecho popular el manejo de presión en diferentes modalidades y el uso de láminas de silicona con buenos resultados ${ }^{24}$, sin embargo, se describen resultados de estudios que evalúan estas estrategias de manera individual y sin estar en combinación con otras terapias. Es por esto, que estudiar el impacto de terapias combinadas y de estrategias que implementen varios mecanismos de acción en un solo dispositivo, genera un aporte importante en la búsqueda de un algoritmo para el manejo de estas lesiones. Se deben establecer criterios que excluyan a pacientes con contraindicaciones, como alergia a los materiales, enfermedades sistémicas que generen hipoperfusión tisular, tejidos infectados o lechos sin granulación³.

El objetivo del presente artículo es describir la efectividad del manejo de cicatrices hipertróficas tempranas por mecanismo de quemadura en una paciente, haciendo uso de una máscara con diseño novedoso elaborada por los profesionales tratantes, que combina el principio de aplicación de presión uniforme por efecto de un biopolímero termoestable, con cubierta interna de silicona.

\section{Caso clínico}

Paciente femenina de 35 años, de raza negra, sin antecedentes de importancia, quien sufre agresión física con agua hirviente generando quemaduras de tercer grado en la unidad estética frontal, periocular, malar bilateral, nasal, labial superior y pabellón auricular derecho. La paciente ingresa a los 17 días después del trauma a la unidad de quemados del Hospital Universitario de Santander (Ver Figura 1a), debido a un retraso en la remisión desde hospital de menor complejidad. Al ingreso la paciente se encuentra son ausencia de signos de infección local o sepsis, con heridas descubiertas limpias y secas. Inicialmente recibe tratamiento con curación de herida interdiaria, realizando desbridamiento mecánico y dejando cobertura con apósitos lubricados con vaselina y sulfadiazina de plata. Además se realizó fisioterapia 2 veces al día, incluyendo ejercicios de estiramiento activos y pasivos con énfasis en las subunidades afectadas. A los 21 días del trauma se obtuvo un adecuado tejido de granulación (Ver Figura 1b). La paciente es llevada a primer manejo quirúrgico 8 días después de su ingreso, 25 días postrauma, injertando con piel de espesor parcial por subunidades estéticas y realizando rafias en los cantos palpebrales para proteger los injertos de piel y para disminuir el riesgo de retracción de los párpados (Ver Figura 2a). Se liberó la cantorrafia a los 15 días posoperatorios (POP), sin embargo, a los 5 días postoperatorios presenta retracción de párpado superior izquierdo (PSI) y ectropión de párpado inferior derecho (PID), a pesar del seguimiento intrahospitalario con continuidad de fisioterapia y lubricación local (Ver Figura 2b). Es llevada a liberación de retracciones y a nuevo injerto de piel de espesor parcial en párpados, bajo protocolo usual de la unidad de quemados con curaciones de la herida y fisioterapia, la cual consistió en ejercicios progresivos de mímica facial con énfasis en el estiramiento del párpado superior, a realizando elevación de las cejas, apertura y oclusión palpebral intermitente, apertura bucal y estiramientos de la unidad de la mejilla con movimientos linguales de la paciente, con asistencia permanente por fisioterapeuta y a tolerancia del paciente. La intervención se complementó con ejercicios para mejorar la capacidad aeróbica y sesiones de terapia ocupacional. 

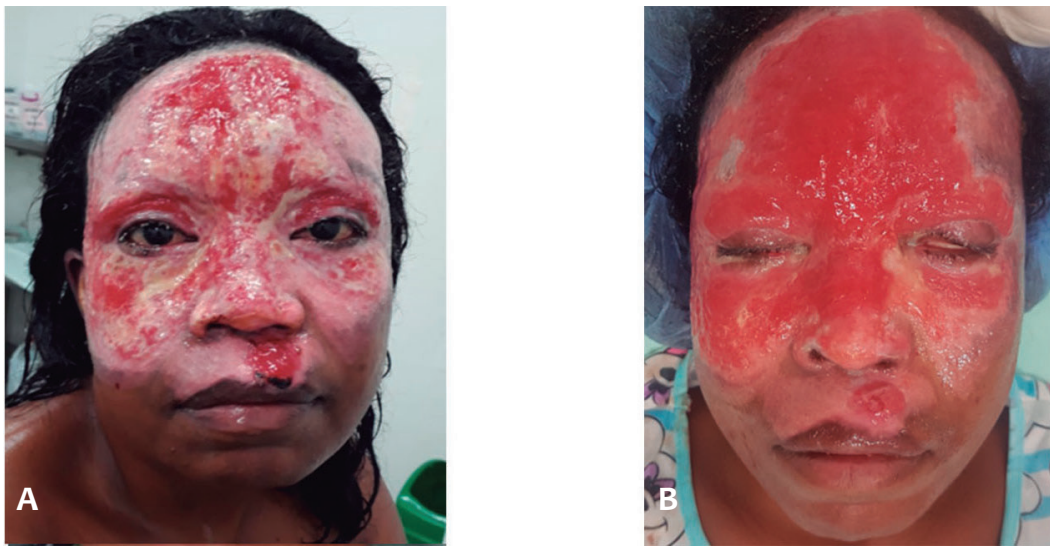

Figura 1. A). Aspecto ingreso 17 días Post Quemadura B). 25 días Post Quemadura

Fuente: autores.
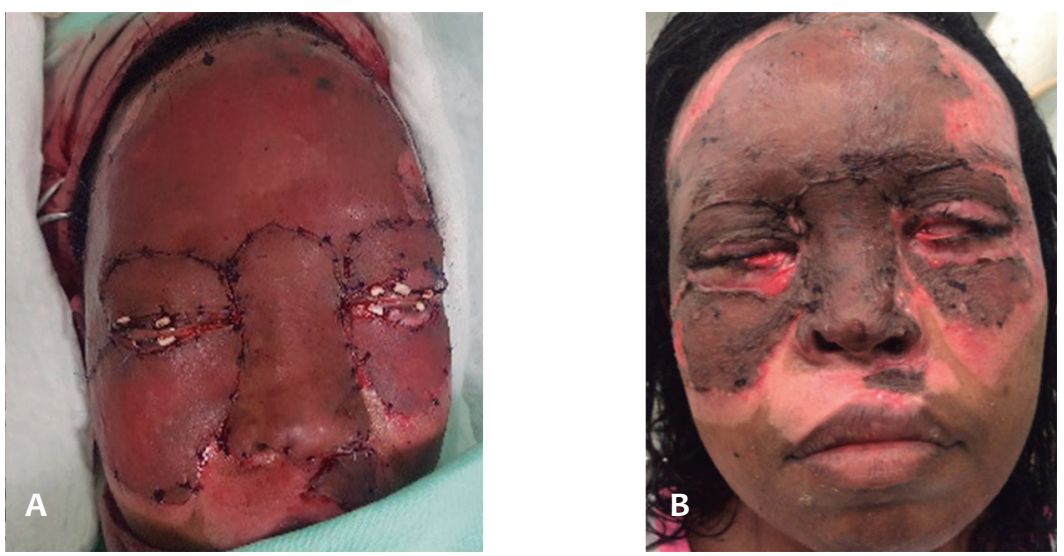

Figura 2. A) POP inmediato IPP B)15 días POP Retracción PSI y Ectropión PID

Fuente: autores.

La paciente tuvo una adecuada evolución de la retracción del PSI y ectropión del PID. Sin embargo, después de dos semanas de terapia, presentó aparición de signos tempranos de cicatrización hipertrófica en las áreas injertadas, dados por aumento en el grosor de la piel, disminución de la elasticidad, bordes irregulares, sobre elevados e hiperpigmentación, obteniendo un puntaje de 12 en la escala de cicatrización de Vancouver (VSS) (Ver Figura 3a). En este punto, se decide en conjunto con el grupo de fisioterapia, la elaboración de una máscara en Aquaplast, polímero termoestable, que se moldea exactamente ajustado al rostro de la paciente y va recubierto internamente con láminas de silicona,las cuales no alteran las características especiales de una piel injertada, más frágil y sensible ${ }^{25,29}$.

Para la elaboración de la máscara, el equipo de la unidad de quemados, realizó un registro fotográfico de la cara y del proceso de evaluación de la cicatriz, aplicando la escala de Vancouver, test de digitopresión, prurito, dolor, movilidad de los músculos de la cara, retracciones y funcionalidad. Se realizó un presupuesto según los materiales disponibles y recursos, ya que la paciente no contaba con recursos económicos para costear materiales como Uvex, Silon, u otros de mayor costo; debiendo garantizar un tratamiento precoz. Adicionalmente, se analizaron los beneficios para la paciente con la utilización de la máscara, el grado de colaboración y aceptación de la misma para su uso. El siguiente paso, fue la preparación de la paciente para la toma de los moldes, que, por su secado rápido requiere de agilidad para evitar el endurecimiento del material, impidiendo la gesticulación del paciente.

Para elaborar el molde de la máscara, se ubicaron gasas estériles sobre la cara de la paciente, para 
Rueda-Gutiérrez JA, Rodríguez-Franco K, Quintero-Moya SL, Gutiérrez-Solano M, Meléndez - Flórez G.

dibujar el contorno de la cara, los ojos, nariz y labio superior. El molde obtenido se transfiere a la lámina de Aquaplast y se calienta con agua a temperatura de $38^{\circ} \mathrm{C}$, que permite la maleabilidad del material. En el momento en el que el material se torna transparente, se retira del agua y se ubica sobre una superficie plana, estéril, cubierta con vaselina para su enfriamiento parcial. Posterior a esto se ubica sobre la cara de la paciente, verificando la posición de los ojos, fosas nasales y bordes de la misma, realizando los ajustes necesarios ejerciendo presión manual leve para contornear adecuadamente las diferentes zonas de la cicatriz que requieren mayor compresión. Una vez realizado el moldeamiento, se retira la máscara de la cara de la paciente y se perfeccionan los bordes de la misma con una pistola de aire caliente, para evitar que zonas irregulares maltraten la piel; así mismo, se determinan las zonas en donde se colocarán los insertos de silicona que, en este caso, correspondían al área de los párpados inferiores y labio superior. Se continúa con la ubicación de tres bandas elásticas de sujeción en seis puntos laterales de la máscara, en los cuales previamente se añadió velcro adhesivo ${ }^{25,26}$ (Ver Figura 3b). El proceso finaliza al aplicar sobre la cara, tanto las láminas de silicona, como la máscara, ajustando las bandas laterales según la tolerancia de la paciente, pero ejerciendo a su vez, presión sobre la cicatriz. Con el ajuste de las bandas, se buscó proporcionar una presión distribuida de manera uniforme y favorecer el contacto de la silicona con las cicatrices. Para terminar, se dieron recomendaciones en cuanto a los cuidados, limpieza y tiempo de uso, el cual debe ser progresivo hasta que la máscara pueda ser tolerada durante 23 horas al día ${ }^{24,28}$, siendo retirada solamente para actividades de alimentación, higiene y terapia física (Ver Figura 3c).
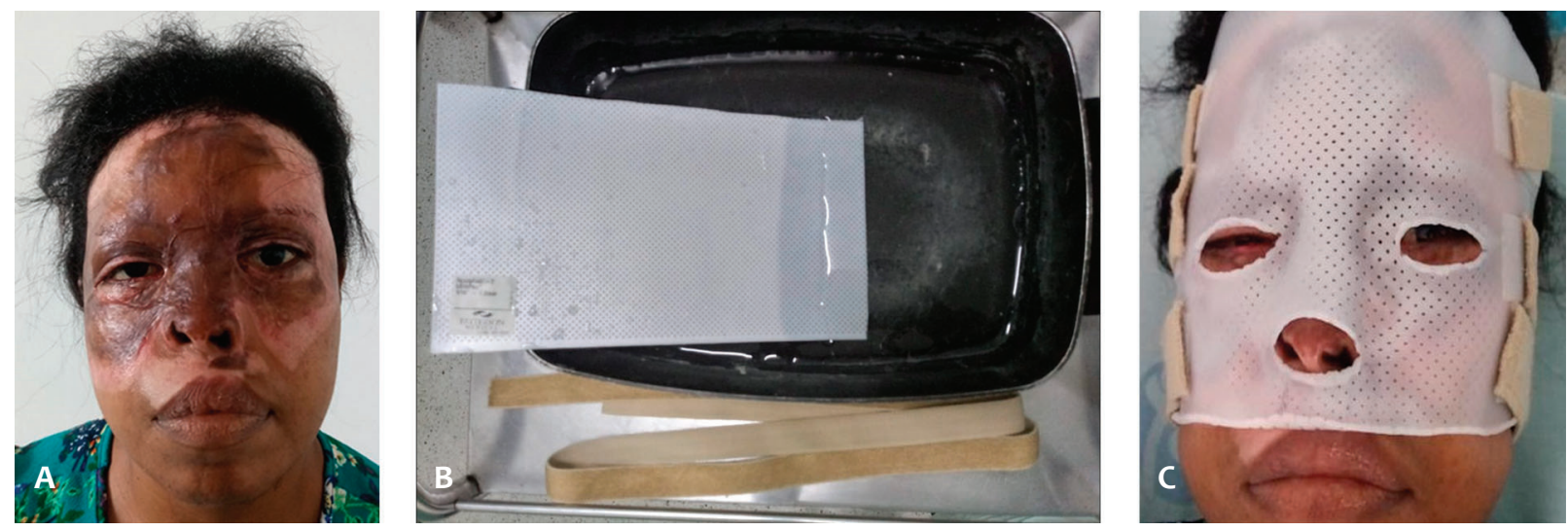

Figura 3 A) 45 días POP. B) Materiales para la elaboración de la Máscara. C) Máscara en posición sobre la paciente

Fuente: Elaboración propia

Adicionalmente, se indica hacer uso estricto de humectantes faciales 3 veces al día, realizando retiro de la máscara y aplicando directamente sobre la piel, junto con medidas de foto-protección UV para evitar la hiperpigmentación y un protocolo riguroso de terapia física y rehabilitación de las zonas injertadas, el cual se inicia a partir de la segunda semana posoperatoria. Las sesiones de fisioterapia fueron de 60 minutos cada una, 2 veces al día, incluyendo masaje de presión progresiva, alternando con estiramientos en el área de los ojos y el labio superior; inicialmente desde zonas adyacentes al injerto einternamente en la boca, para posteriormente avanzar hacia una presión directa sobre la cicatriz. Los 20 minutos restantes, consistieron en ejercicios activos de estiramiento a través de la mímica facial, combinando movimientos, y la educación a la paciente. Inmediatamente termina la sesión, el fisioterapeuta ubica las láminas de silicona sobre las cicatrices y coloca nuevamente la máscara, ajustando diariamente las bandas laterales, de manera progresiva, para aumentar la presión.

La paciente continúa el plan de cuidado en su hogar, haciendo uso de la máscara la mayor parte del día, con un mínimo de 16 horas, incluyendo ejercicios de estiramiento y masaje de presión, logrando una mejoría significativa en el color, grosor, flexibilidad, altura y textura de su cicatriz, así como ausencia de retracciones cicatrízales, lagoftalmías o ectropión, tanto en estado de reposo como en la evaluación dinámica, obteniendo un puntaje de 2 en la VSS a los 6 meses de seguimiento (Ver Figura 4). 

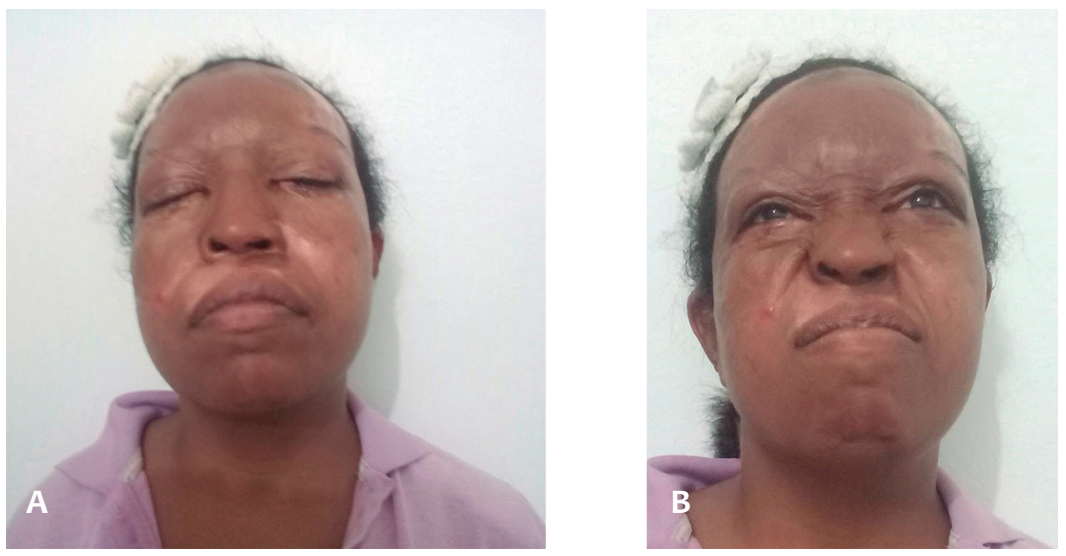

Figura 4. 6 meses POP A) Estático B) Dinámico

Fuente: autores.

\section{Discusión}

La diana terapéutica de los diferentes tratamientos se encuentra dirigida a la regulación de los fenómenos de fibroplasia, granulación, contracción y epitelización, que hacen parte de la fase proliferativa de la cicatrización $n^{17}$; y adicionalmente, impactar en la arquitectura y su remodelación, garantizando que no se depositen mayores cantidades de matriz extracelular con colágeno denso y menos funcional que el de la piel normal'15,16,28.

Se ha visto menor incidencia de cicatrización hipertrófica y sus secuelas retráctiles en los casos en que la quemadura afecta áreas especiales como cara, manos y articulaciones, siempre que los injertos se realicen antes de comenzar la segunda semana ${ }^{18}$.

El objetivo de acompañar el proceso de cicatrización con terapias es minimizar las diferentes complicaciones que deja el crecimiento desorganizado de las fibras de colágeno, como son: las limitaciones funcionales para la comunicación, alimentación, respiración, autocuidado, aspecto cosmético y sentido de identidad.

El masaje sobre la cicatriz en proceso de maduración, con duración de al menos 5 minutos, funciona para disminuir la tensión de los tejidos, activar los mecanoreceptores que juegan un papel importante en la activación de la apoptosis y permitir que se produzca una arquitectura más ordenada ${ }^{28}$.

Por su parte, la aplicación de las láminas de silicona o geles que la contengan, se basa en la teoría de la generación de electricidad estática que hace disminuir la producción de fibroblastos y mastocitos, así como inducir la producción de enzimas como colagenasa y metaloproteinasas. La literatura también describe su potencial a la hora de mantener la hidratación del estrato córneo, lo que agiliza los procesos de cicatrización ${ }^{27}$. La presentación de la silicona en láminas facilita la aplicación y recambio, en particular, en zonas especiales como rostro y en pequeñas áreas móviles. También tiene indicación en zonas extensas que tengan predisposición a la cicatrización hipertrófica, para cuyo caso, las prendas de presión representan una opción adicional para ejercer presión localizada ${ }^{30}$.

La aplicación individual de cada alternativa ha generado estudios de prevención y tratamiento, incluidos en meta-análisis como el que incluye 20 ensayos que compararon la cubierta de gel de silicona adhesiva con ningún tratamiento; el apósito sin silicona; otros productos de silicona; la terapia láser; la inyección de acetónido de triamcinolona; el extracto de cebolla tópico y la terapia de presión. En los estudios de prevención, cuando se comparó con una opción de ningún tratamiento, la cubierta de gel de silicona redujo la incidencia de cicatrices hipertróficas en los pacientes propensos a cicatrices (riesgo relativo [RR] 0,46; intervalo de confianza [IC] del 95\%: 0,21 a 0,98). En los estudios de tratamiento, la cubierta de gel de silicona produjo una reducción estadísticamente significativa del grosor de la cicatriz (diferencia de medias [DM] -2,00; IC del 95\%: -2,14 a $-1,85$ ) y una mejoría del color (RR 3,49; IC del 95\%: 1,97 a 6,15$)^{31}$.

En cuanto a la aplicabilidad de terapia de compresión después de la escisión del tejido hipertrófico, 
se encuentran estudios prospectivos de casos y controles, con una tasa de no recurrencia de 70.5 a 95 por ciento3 ${ }^{3}$ similar a la terapia de vendaje oclusivo. La terapia de compresión tiene los mejores resultados si el dispositivo de presión se coloca durante al menos 12 horas por día para al menos seis meses de una presión de al menos 24 mmHg32.

La combinación de la máscara con los insertos de silicona se establece con el fin de dar mayor presión a las cicatrices que se están hipertrofiando, teniendo en cuenta además los efectos de la silicona que ya fueron descritos inicialmente, y aunque no hay evidencia concluyente al respecto, los resultados de combinar dos mecanismos son claramente observables ${ }^{22,27}$.

Sin embargo, no se ha encontrado literatura que haga aplicación de esta técnica de forma concomitante o coadyuvante con otra, sino como terapia única.

Dentro de las diferentes estrategias se ha estudiado ampliamente la eficacia de la presoterapia que, al generar un ambiente hipoxémico, disminuye la proliferación celular e induce apoptosis. Adicionalmente, genera un efecto mecánico que disminuye el volumen cicatricial hasta en un $15 \%$, al permitir una mejor organización de los nuevos haces de colágeno. Su aplicabilidad tiene resultados cuando se supera la presión capilar, que en un promedio es de 20 a $30 \mathrm{mmHg}^{17}$.

Se considera el tiempo de uso diario en un promedio de 16 a 24 horas, retirándose únicamente para la higiene, humectación y realizar terapia física, basado en la hipótesis de que el flujo sanguíneo disminuye por la presión, reduciendo así la síntesis de colágeno como resultado. Además de producir hipoxia, también degenera la estructura y cúmulo de las fibrillas de colágeno. Se puede apreciar que la pigmentación roja de las cicatrices disminuye al neutralizar la vascularización, así como el aplanamiento de abultamientos y disminución de retracciones ${ }^{25,33}$.

No hay consenso sobre la cantidad necesaria de presión, por lo que se ha manejado un rango de presión aplicada entre 23 y $25 \mathrm{mmHg}$, pareciendo efectivo para superar la presión capilar en el tejido cicatricial; sin embargo, estos valores varían según la literatura ${ }^{29,33}$.

El objetivo de la terapia de compresión es mejorar los síntomas propios de la cicatriz, como el enrojecimiento. La finalidad terapéutica es inducir la maduración de la cicatriz quirúrgica. En la literatura existen estudios en donde se describe la aplicación de máscaras con distribución de presión uniforme sobre las cicatrices producidas por quemaduras, monitorizando la presión ejercida mediante sensores. El inicio de las terapias será entre la semana 3 y el mes 6 después de una lesión o cirugía, con resultados variables en la Escala POSAS (Escala de Valoración Objetiva Paciente y Observador) en pacientes con hipertrofia de cicatrices, por lo que se sugiere un inicio más precoz de la aplicación de la terapia compresiva ${ }^{23,35}$.

Es por esto que resulta innovador hacer uso concomitante de dos principios que supondrían un mayor control sobre los procesos involucrados en la hipertrofia de las cicatrices. Park describe la implementación de un dispositivo denominado "Magsil" para tratar cicatrices hipertróficas en lóbulos auriculares, el cual consiste en 2 imanes con cubierta de silicona que, al ser posicionados en la superficie anterior y posterior de la cicatriz y atraídos por la fuerza magnética, produce una presión constante que se combina con los efectos de la silicona, obteniendo buenos resultados, pero con aplicación limitada únicamente a la región auricular ${ }^{35}$. Este concepto fue aplicable en el dispositivo aplicado a nuestra paciente permite tratar de manera efectiva, a través de diferentes mecanismos de acción, las cicatrices hipertróficas localizadas en una zona anatómica especial, con una superficie extensa y por medio de un único dispositivo, el cual es removible y fácil de usar, favoreciendo la adherencia terapéutica ${ }^{6}$.

La valoración de la evolución de la cicatriz se hace mediante la evaluación de características como el color (eritema y pigmentación), flexibilidad, grosor e irregularidad, las cuales se pueden consignar a través de diferentes escalas como la escala de cicatriz de Vancouver (VSS) y la evaluación de la cicatriz del observador (POSAS), ampliamente utilizados en la práctica clínica debido a su facilidad de uso; sin embargo, con limitada reproducibilidad por ser observador-dependientes ${ }^{14,36}$.

En este caso se evidenció una mejoría clínica por control del prurito y el dolor, además del aumento de la flexibilidad, reducción en la pigmentación y espesor de los tejidos abordados, logrando una disminución de 10 puntos en la escala VSS ${ }^{38}$.

La duración del tratamiento depende del grado de hipertrofia observado y se encuentra a criterio del 
equipo médico tratante. Se recomienda realizar el seguimiento clínico mensualmente para evaluar la evolución de las cicatrices; en promedio, la duración total del tratamiento es de seis meses y en algunos casos puede prolongarse hasta por dos años ${ }^{18}$. Si no se evidencia mejoría objetiva con la primera línea de tratamientos tópicos no invasivos o si se observa una progresión hacia cicatriz queloide, se puede considerar la siguiente fase de manejo, que consiste en inyecciones intralesionales de corticosteroides, terapia con láser, escisión quirúrgica tardía, uso de postoperatorias 5-fluorouracilo (5-FU), imiquimod, entre otros ${ }^{14,40,41}$.

Se han realizado estudios que buscan además de manejar, prevenir el desarrollo de las cicatrices hipertróficas, evaluando la eficacia profiláctica de algunos materiales y fármacos. Un estudio prospectivo, utiliza el acetato de dexametasona en la prevención de la hiperplasia cicatricial. Se halló una disminución estadísticamente significativa al comparar la presencia de hiperplasia cicatricial entre ambas lesiones, una que recibió infiltración en el tejido previo a una incision controlada $(p=0.06)$ y otra sin dicha intervención $(p=0.02)$, al hacer un seguimiento postoperatorio de 6 a 15 meses $^{40}$.

Así mismo, se encuentra en experimentación la administración de avotermina previo a la activación de cascadas de factores de crecimiento involucrados en la cicatrización de tejidos con incisiones programadas. En teoría, este factor de crecimiento transformante $\beta_{3}$, al ser morfogénico cutáneo, actúa regulando las cascadas de señalización con un efecto modulador positivo"1.

Lo anterior deja entrever el advenimiento de múltiples y novedosas alternativas, materiales, fármacos biológicos y terapias experimentales combinadas, con resultados prometedores.

\section{Conclusiones}

El uso de la máscara facial diseñada permite aplicar presión distribuida de manera homogénea sobre toda la región facial y un contacto permanente de la silicona sobre las cicatrices; es cómoda, ligera y removible, favoreciendo la adherencia del paciente al tratamiento. Cuando se combina con un estricto protocolo de fisioterapia, se promueve el adecuado proceso de maduración cicatricial, permitiendo dar un tratamiento satisfactorio a pacientes con
Máscara termoplástica recubierta de silicona para el manejo de la cicatriz hipertrófica facial

cicatrices hipertróficas secundarias a quemaduras faciales severas.

Se sugiere la realización de más estudios que evalúen la efectividad de esta terapia en pacientes con secuelas de quemaduras faciales, que puedan replicar los resultados obtenidos en este caso.

Finalmente, se recomenda que ante un caso de quemadura secundaria a agresión física se oriente a la víctima sobre el tratamiento adecuado, en el lugar apropiado, con el personal especializado para tal fin. Además de orientar al paciente sobre las conductas sociales pertinentes como denunciar al agresor, evitar exponerse nuevamente a otra agresión, dar a conocer la nueva ley al respecto y buscar la ayuda médica, psicológica, social y legal correspondiente en cada caso.

\section{Agradecimientos}

Extendemos un agradecimiento especial al grupo de terapia física, terapia ocupacional y enfermería, de la Unidad de Quemados del Hospital Universitario de Santander, su labor, dedicación, amor y compromiso con los pacientes es indispensable para su recuperación y hace más llevadero este proceso.

\section{Referencias bibliográficas}

1. Das KK, Olga L, Peck M, Morselli PG, Salek AJM. Management of acid burns: Experience from Bangladesh. Burns. 2015;41(3):484492.

2. Acid Survivors Foundation (ASF). Annual Progress Status of ASF [Internet]. Mirpur: Acid Survivors Foundation; 2017. Disponible en: http://old.acidsurvivors.org/images/frontImages/Annual_ Report 2016 24.07.2017.pdf

3. Welsh J. "It was like burning in hell": a comparative exploration of acid attack violence [tesis de maestría]. Chapel Hill: Carolina papers on international health, Universidad de Carolina del Norte; 2009.

4. Guerrero L. Burns due to acid assaults in Bogotá, Colombia. Burns. 2013;39(5):1018-23.

5. Ley por medio de la cual se crea el artículo $116 \mathrm{~A}$, se modifican los artículos 68A, 104, 113, 359 y 374 de la ley 599 de 2000 y se modifica el artículo 351 de la ley 906 de 2003. (Diario oficial N. 49747.6, número 1773, 6 de enero de 2016)

6. Gaviria JL, Santamaria N, Velandia C, Balanta C, Quintero A. Georreferenciación de las quemaduras en Bogotá, Colombia. Rev Colomb Cir Plást Reconstr. 2019;25(2):61-71.

7. Bombaro KM, Engrav LH, Carrougher GJ, Wiechman SA, Faucher $\mathrm{L}$, Costa BA et al. What is the prevalence of hypertrophic scarring following burns?. Burns. 2003;29(4):299-302.

8. Kwan P, Desmoulière A, Tredget EE. Molecular and Cellular Basis of Hypertrophic Scarring. En: Herndon DN, editores. Total Burn Care. $4^{\mathrm{a}}$ ed. Edinburgh: Elsevier; 2018. p. 455-465.

9. Gauglitz GG, Korting HC, Pavicic T, Ruzicka T, Jeschke MG. Hypertrophic scarring and keloids: pathomechanisms and current and emerging treatment strategies. Mol Med. 2011;17(12):113-125. 
10. Ogawa R, Akaishi S. Endothelial dysfunction may play a key role in keloid and hypertrophic scar pathogenesis - keloids and hypertrophic scars maybe vascular disorders. Med Hypotheses. 2016;96:51-60.

11. Ferguson MJ, Duncan J, Bond J, Bush J, Durani P, So K, et al. Prophylactic administration of avotermin for improvement of skin scarring: three double-blind, placebo-controlled, phase I/II studies. Lancet. 2009;373(9671):1264-74.

12. Villegas Alzate F. Cirugía plástica para el médico general, estudiantes de la salud y otros profesionales. $2^{\mathrm{a}}$ ed. Bogotá, Colombia: Ecoe Ediciones;2019.

13. Micallef L, Vedrenne N, Billet F, Coulomb B, Darby IA, Desmoulière A. The myofibroblast, multiple origins for major roles in normal and pathological tissue repair. Fibrogenesis Tissue Repair. 2012;5(Suppl 1):S5.

14. Lee KC, Bamford A, Gardiner F, Agovino A, Ter Horst B, Bishop $\mathrm{J}$, et al. Burns objective scar scale (BOSS): Validation of an objective measurement devices based burn scar scale panel. Burns. 2020;46(1):110-20.

15. Xue M, Jackson CJ. Extracellular Matrix Reorganization During Wound Healing and Its Impact on Abnormal Scarring. Adv Wound Care (New Rochelle). 2015;4(3):119-36.

16. Hinz B. The role of myofibroblasts in wound healing. Curr Res Transl Med. 2016;64(4):171-7.

17. To WS, Midwood KS. Plasma and cellular fibronectin: distinct and independent functions during tissue repair. Fibrogenesis Tissue Repair. 2011;4:21.

18. Cubison TC, Pape SA, Parkhouse N. Evidence for the link between healing time and the development of hypertrophic scars (HTS) in paediatric burns due to scald injury. Burns. 2006;32(8):992-9.

19. Herranz P, Santos X. Cicatrices, guía de valoración y tratamiento. Madrid: Meda Pharma; 2013.

20. Hospital San Vicente Fundación de Medellín y Physician for Peace. Experiencias Tercer Encuentro Unidades de Quemados de Colombia. Medellín: 2018.

21. Thompson CM, Sood RF, Honari S, Carrougher GJ, Gibran NS. What score on the Vancouver Scar Scale constitutes a hypertrophic scar? Results from a survey of North American burn-care providers. Burns. 2015;41(7):1442-1448.

22. Tsuge T, Aoki M, Akaishi S, Dohi T, Yamamoto H, Ogawa R. Geometric modeling and a retrospective cohort study on the usefulness of fascial tensile reductions in severe keloid surgery. Surgery. 2020;167(2):504-509.

23. Gee Kee EL, Kimble RM, Cuttle L, Stockton KA. Scar outcome of children with partial thickness burns: A 3 and 6 month follow up. Burns. 2016;42(1):97-103.

24. Bao Y, Xu S, Pan Z, Deng J, Li X, Pan F, et al. Comparative Efficacy and Safety of Common Therapies in Keloids and Hypertrophic Scars: A Systematic Review and Meta-analysis. Aesthetic Plast Surg. 2019;44(1):207-218.

25. Colla C, Kant SB, Van den Kerckhove E, Van der Hulst RR, Piatkowski de Grzymala AA. Manual fabrication of a specialized
MÉD.UIS. 2020;33(3):49-58

transparent facial pressure mask: A technical note. Prosthet Orthot Int. 2019;43(3):356-360.

26. Kant S, Colla C, Kerckhove E, Piatkowski de Grzymala A. Clinical effects of transparent facial pressure masks: A literature review. Prosthet Orthot Int. 2019;43(3):349-55.

27. Grabowski G, Pacana MJ, Chen E. Keloid and Hypertrophic Scar Formation, Prevention, and Management: Standard Review of Abnormal Scarring in Orthopaedic Surgery. J Am Acad Orthop Surg. 2020;28(10):e408-e414.

28. Nedelec B, Carter A, Forbes L, Chen Hsu SC, McMahon M, Parry I, et al. Practice guidelines for the application of nonsilicone or silicone gels and gel sheets after burn injury. J Burn Care Res. 2015;36(3):345-374.

29. Hu MS, Zielins ER, Longaker MT, Lorenz HP. Scar prevention, treatment, and revision. En: Gurtner GC, Neligan PC, editors Plastic Surgery. Philadelphia: Elsevier; 2018. p. 196-213.

30. O'Brien L, Jones DJ. Silicone gel sheeting for preventing and treating hypertrophic and keloid scars. Cochrane Database Syst Rev. 2013(9):CD003826.

31. Hernandez C, Toro A. Approach and management of hypertrophic scars and keloids. Rev Asoc Colomb Dermatol. 2011;19: 218-28.

32. Mokos ZB, Jović A, Grgurević L, Dumić-Čule I, Kostović K, Čeović R, et al. Current Therapeutic Approach to Hypertrophic Scars. Front. Med. 2017;4(83).

33. Kant SB, Colla C, Van den Kerckhove E, Van der Hulst RRWJ, Piatkowski de Grzymala A. Satisfaction with Facial Appearance and Quality of Life after Treatment of Face Scars with a Transparent Facial Pressure Mask. Facial Plast Surg. 2018;34(4):394-9.

34. Eming SA. Biology of wound healing. In: Bolognia JL, Schaffer JV, Cerroni L, editors. Dermatology. 4a ed. Philadelphia, PA: Elsevier; 2018. p. 2413-24.

35. Park TH. New pressure device, "Magsil," as an adjuvant pressure therapy for ear keloids. Arch Facial Plast Surg. 2012;14(4):298-9.

36. Oliveira GV, Chinkes D, Mitchell C, Oliveras G, Hawkins HK, Herndon DN. Objective assessment of burn scar vascularity, erythema, pliability, thickness, and planimetry. Dermatol Surg. 2005;31(1):48-58

37. Esselman P, Kowalske K. Burn Rehabilitation, An Issue of Physical Medicine and Rehabilitation Clinics, Volume 22-1. En: Physical Medicine and Rehabilitation Clinics of North America. 1a ed. 2011. p. 201-357.

38. Shirakami E, Yamakawa S, Hayashida K. Strategies to prevent hypertrophic scar formation: a review of therapeutic interventions based on molecular evidence. Burns Trauma. 2020 Jan 2;8:tkz003.

39. El Ayadi A, Jay JW, Prasai A. Current Approaches Targeting the Wound Healing Phases to Attenuate Fibrosis and Scarring. Int J Mol Sci. 2020 Feb 7;21(3):1105

40. Villafuerte-Vélez CK, Castro-Cabrera AC, Restrepo-Villafuerte C. Trans-surgery prophylactic corticotherapy of cicatricial hyperplasia. Clinic-stadistic prospective study. Cir. plást. Iberolatinoam.2017;43(1):33-9. 